\title{
Bestimmung von Carbonsäuren aus wäßriger Lösung mittels Kapillar-Gas-Chromatographie
}

\section{R. Gloor / H. Leidner}

Eidg. Anstalt für Wasserversorgung, Abwasserreinigung und Gewässerschutz (EAWAG), CH-8600 Dübendorf - Zürich, Schweiz

\section{Summary}

\section{- Determination of Carboxylic Acids in Aqueous Solution} by Capillary Gas Chromatography

$A$ method is presented for the determination of carboxylic acids at low concentrations (ppb-range, $C_{1}-C_{24}$ carboxylic acids) in aqueous solution by capillary gas chromatography. Practical examples are given by the analysis of a pheny/carboxylic acid mixture and samples of waste water and digested sludge water.

\section{Zusammenfassung}

Es wird eine Vorschrift für die Bestimmung niedriger Carbonsäurekonzentration ( $p p b^{*}$-Bereich, $C_{1}-C_{24}$ Carbonsäuren) aus wäßrigem Milieu mittels Kapillar-GasChromatographie gegeben. Die praktische Anwendung wird an einer Pheny/carbonsäuremischung, einer Abwasserund Faulschlammwasserprobe gezeigt.

\section{Einleitung}

Die gas-chromatographische Bestimmung organischer Säuren aus wäßriger Lösung läßt sich je nach Fragestellung und Säurenspektrum auf verschiedene Arten durchführen:

a) Direkte Applikation der wäßrigen Lösung [1,2].

b) Isolieren der Säuren aus der wäßrigen Lösung, Herstellung flüchtiger Derivate und anschließende gaschromatographische Analyse.

Krupčik et al. [3] beschreiben zwar die direkte kapillargas-chromatographische Analyse $\operatorname{der} \mathrm{C}_{2}-\mathrm{C}_{6}$-Säuren und ihrer Isomeren (Säule: Ucon-LB-550-X + Phosphorsäure), jedoch ist unseres Wissens bis jetzt die Anwendung wäßriger Lösungen nur auf gepackten Säulen möglich und auf die Bestimmung der niedermolekularen Glieder dieser Verbindungsklasse von $\mathrm{C}_{1}$ bis $\mathrm{C}_{6}$ beschränkt.

Um ein breiteres Carbonsäurenspektrum erfassen $\mathbf{z u}$ können und die Vorteile der Kapillar-Gas-Chromatographie

\footnotetext{
* $1 \mathrm{ppb}=1 \mu \mathrm{g}$ in $1 \mathrm{dm}^{3}$
}

(hohe Auflösung, größere Empfindlichkeit [4]) auszunutzen, ist eine Überführung in leichtflüchtige Derivate - im allgemeinen Ester - notwendig. Die klassischen Veresterungsmethoden mit überschüssigem Alkohol und einer Säure $\left(\mathrm{HCl}, \mathrm{H}_{2} \mathrm{SO}_{4}, \mathrm{BF}_{3}\right)$ als Katalysator $[4,5]$ sind im Mikromaßstab schwierig durchzuführen und ohne weitere Aufbereitung für anschließende Kapillar-GasChromatographie auch ungeeignet.

Huwyler [7] beschreibt eine Veresterungsmethode, nach der ng-Mengen Carbonsäure mit Diazomethan in ihre Methylester überführt werden können. Gemäß Angaben ist dieses Verfahren jedoch nur für die Veresterung der Carbonsäuren $\mathrm{C}_{6}-\mathrm{C}_{12}$ geeignet.

Ebenfalls für die gas-chromatographische Analyse geeignete Carbonsäurederivate sind die Trimethylsilylester, besonders wenn eine massensprektrometrische Identifikation erforderlich ist. Da das Silylierungsreagenz jedoch nicht selektiv mit der Carboxylgruppe reagiert (Hydroxylgruppen werden miterfaßt), ist meist eine Vortrennung notwendig [8]. Außerdem sind die Trimethylsilylester extrem feuchtigkeitsempfindlich, was ihre Handhabung zusätzlich erschwert.

Gehrke und Goerlitz [9] beschreiben ein einfaches Verfahren für die Bestimmung von Fettsäuren in biologischem Material. Dabei werden die Fettsäuren über die Silbersalze mit Alkylhalogenid in ihre Ester überführt. Wir entschieden uns aus folgenden Gründen für diese Methode und entwickelten sie für die Anwendung im $\mu$ g-Bereich:

1. Das Trocknen der Probe geschieht verlustlos über die Silbersalze der Säuren.

2. Die Veresterung kann in Pentan durchgeführt werden (ideales Lösungsmittel für die Kapillar-Gas-Chromatographie).

3. Die Carboxylgruppen werden selektiv erfaßt.

Die analytische Zuverlässigkeit der Methode prüften wir an den $\mathrm{C}_{1}-\mathrm{C}_{24}$-Carbonsäuren.

\section{Methoden}

\section{Arbeitsvorschrift}

$5 \mathrm{~cm}^{3}$ wäßrige Probe werden in einem $100 \mathrm{~cm}^{3}$ Rundkolben (mit Sovirelverschluß) mit $1 \mathrm{n} \mathrm{KOH}$ auf $\mathrm{pH}=$ 10-11 eingestellt. Anschließend werden mit $1 \mathrm{~m} \mathrm{AgNO}_{3}$ Lösung $\left(\mathrm{AgNO}_{3}\right.$ p.a., Merck) die Silbersalze der Carbonsäuren ausgefällt und ein Überschuß $\mathrm{AgNO}_{3}$-Lösung bis 


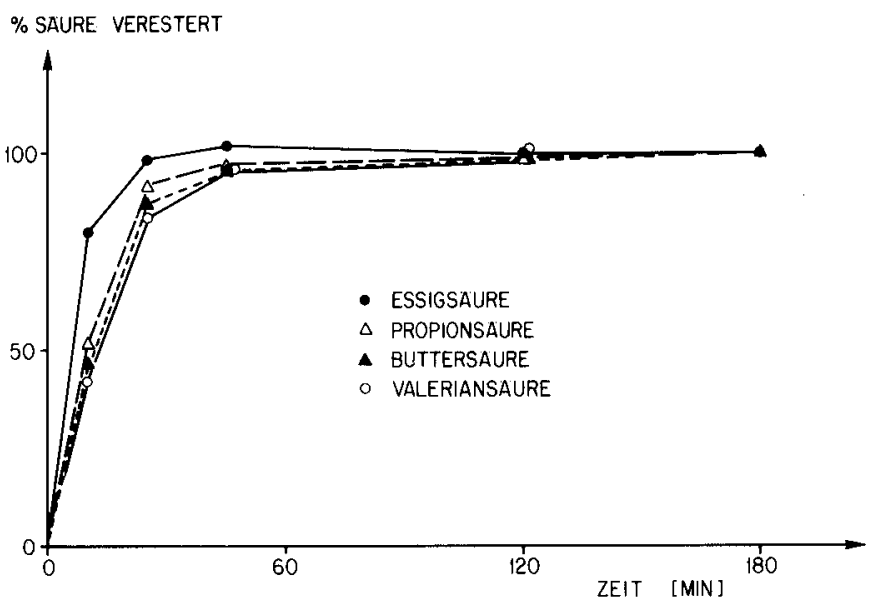

Fig. 1

Kinetik der Veresterungsreaktion von Carbonsäuresilbersalzen mit Äthyljodid.

Kinetics of esterification with ethyl iodide of silver salts of carboxylic acids.

$\mathrm{zu}$ einer freien $\mathrm{Ag}^{+}-$Konzentration von $10^{-3}$ bis $10^{-2}$ $\mathrm{Mol} / \mathrm{dm}^{3}$ zugefügt. Dieser Wert wurde empirisch ermittelt und kann mittels Potentialmessung (Silber gegen Kalomel $+410 \mathrm{mV}$ ) reproduzierbar eingestellt werden. Gemäß experimentellen Befunden muß der $\mathrm{Ag}^{+}$-Endkonzentration sorgfältige Beachtung zukommen, da bei geringerer $\mathrm{Ag}^{+}$. Konzentration die Veresterung im nachfolgenden Schritt unvollständig verläuft, während höhere $\mathrm{Ag}^{+}-$Konzentrationen vermehrt zu Nebenreaktionen führen. Nach Zugabe von etwas geglühtem Quarzsand (Rührhilfe bei der Veresterungsreaktion) wird gefriergetrocknet oder am Rotationsverdampfer zur Trockene eingeengt. Der Gefriertrocknung ist jedoch der Vorzug zu geben, da bei diesem Verfahren eine Krustenbildung vermieden wird.

Der Trockenrückstand wird zur Veresterung mit $2,5 \mathrm{~cm}^{3}$ Pentan - enthaltend $5 \mathrm{ppm}$ n-Tridecan als internen Standard - und 5-10 $\mathrm{mm}^{3}$ A thyljodid (Äthyljodid puriss. p.a. Fluka, 2mal destilliert) in dicht verschlossenem Kolben während 3 Stunden im Dunkeln bei $\sim 10{ }^{\circ} \mathrm{C}$ kräftig geschüttelt (Proben ständig im Dunkeln aufbewahren). Die Pentanlösung kann direkt gas-chromatographisch analysiert werden.

Kinetische Untersuchungen der Veresterungsgeschwindigkeit von Essigsäure, Propionsäure, Buttersäure und Valeriansäure (Fig. 1) ergaben, daß für diese Komponenten bereits nach 60 Minuten Kontaktzeit der Endwert erreicht ist. Für Proben unbekannter Zusammensetzung sollten jedoch aus Sicherheitsgründen 3 Stunden Reaktionsdauer eingehalten werden.

Aus praktischen Gründen sowie analytischen Überlegungen (Beschränkung von Störeinflüssen auf ein Minimum, z. B. von Fremdsalz) gelten 5-20 $\mathrm{cm}^{3}$ Probenvolumen als geeignet. Für die präparative und analytische Weiterverarbeitung der gefriergetrockneten Probe sollten wenigstens 1-10 $\mu \mathrm{g}$ je Säurekomponente vorliegen. Das bedeutet, daß Proben mit einer Säurekonzen- tration von $0,1 \mathrm{ppm}$ direkt gemäß Vorschrift verarbeitet werden können, bei geringeren Konzentrationen jedoch eine Anreicherung erforderlich ist.

\section{Gas-Chromatographie}

Die gas-chromatographischen Analysen wurden mit einem Fractovap Mod. GJ (Fa. Carlo Erba, Mailand) mit Injektorteil nach Grob [4] und FI-Detektor auf Glaskapillarsäulen (Bezugsquelle H. und G. Jaeggi, CH-9043 Trogen) durchgeführt.

Als geeignete Trennkapillaren erwiesen sich eine UconHB 5100-Säule ( $50 \mathrm{~m}, 0,31 \mathrm{~mm}$ i- $\varphi$ ) für die $\mathrm{C}_{1}-\mathrm{C}_{13^{-}}$ Carbonsäure-Ä thylester (Fig. 2) und eine weniger polare, höher heizbare SE 54-Säule $(20 \mathrm{~m}, 0,38 \mathrm{~mm} \mathrm{i}-\emptyset)$ für die $\mathrm{C}_{4}-\mathrm{C}_{24}$-Carbonsäure-Ä thylester (Fig. 3).

Die Identifikation der Peaks wurde mit Reinsubstanzen und Massenspektrometrie durchgeführt, und die Auswertung der Chromatogramme erfolgte mit einem elektronischen Integrator (Mod. CRS $100 \mathrm{~A}$, Infotronics).

\section{Wiederholbarkeit und Richtigkeit der Methode}

Von einer wäßrigen Stammlösung folgender Carbonsäuren: Essigsäure (19.6 ppm), Propionsäure (20.8 ppm), Isobuttersäure (17.0 ppm), Buttersäure (22.0 ppm), 2-Methylbuttersäure (22.2 ppm), Valeriansäure (16.4 ppm) wurden in 4 Versuchsansätzen je $5 \mathrm{~cm}^{3}$ nach der oben gegebenen Veresterungsvorschrift verarbeitet und mittels GasChromatographie quantitativ [11] bestimmt (Tab. I). Die aus den 4 Versuchen erhaltenen Werte weisen eine gute Übereinstimmung auf, und ihr Mittelwert liegt im Vergleich mit dem theoretischen Wert durchaus im Rahmen der mittels Gas-Chromatographie erreichbaren Richtigkeit.

\section{Praktische Anwendung}

\section{a) Kommunales Abwasser}

Aufgrund von Voruntersuchungen und Literaturangaben $[12,13]$ sind die Carbonsäuren in häuslichem Abwasser im Bereich von wenigen ppb* bis zu einigen ppm zu erwarten. Um auch die Glieder mit geringer Konzentration erfassen zu können, ist zunächst eine Anreicherung erforderlich.

$250 \mathrm{~cm}^{3}$ eines kommunalen, mechanisch vorgeklärten $\mathrm{Ab}$ wassers (Stadt Zürich) werden nach Zentrifugation $(30000 \mathrm{~g} / 15 \mathrm{~min})$ und Filtration über ein Membranfilter $(0,45 \mu \mathrm{m}$ Porenweite $) \mathrm{mit} \mathrm{KOH}$ bis $\mathrm{pH} 11$ versetzt und nach 30 Minuten Kochen am Rückfluß gefriergetrocknet. Nach Extraktion des Trockenrückstandes mit Methylenchlorid (Entfernung störender Begleitstoffe) werden die Carbonsäuren unter Eiskühlung mit salzsaurem Äther vom anorganischen Rückstand abgetrennt, durch Zugabe von $\mathrm{KOH}$ in wäßrige Phase übergeführt und nach Vorschrift verestert (Fig. 4a, 4b und Tab. II). Gemäß Untersuchungen zur Wiederholbarkeit ( 5 Versuche) des für Abwasser beschriebenen Aufbereitungsverfahrens ergab sich bei folgenden geprïften Säuren (Ameisen-, Essig-, Propion-,

* $\quad 1 \mathrm{ppb}=1 \mu \mathrm{g}$ in $1 \mathrm{dm}^{3}$ 


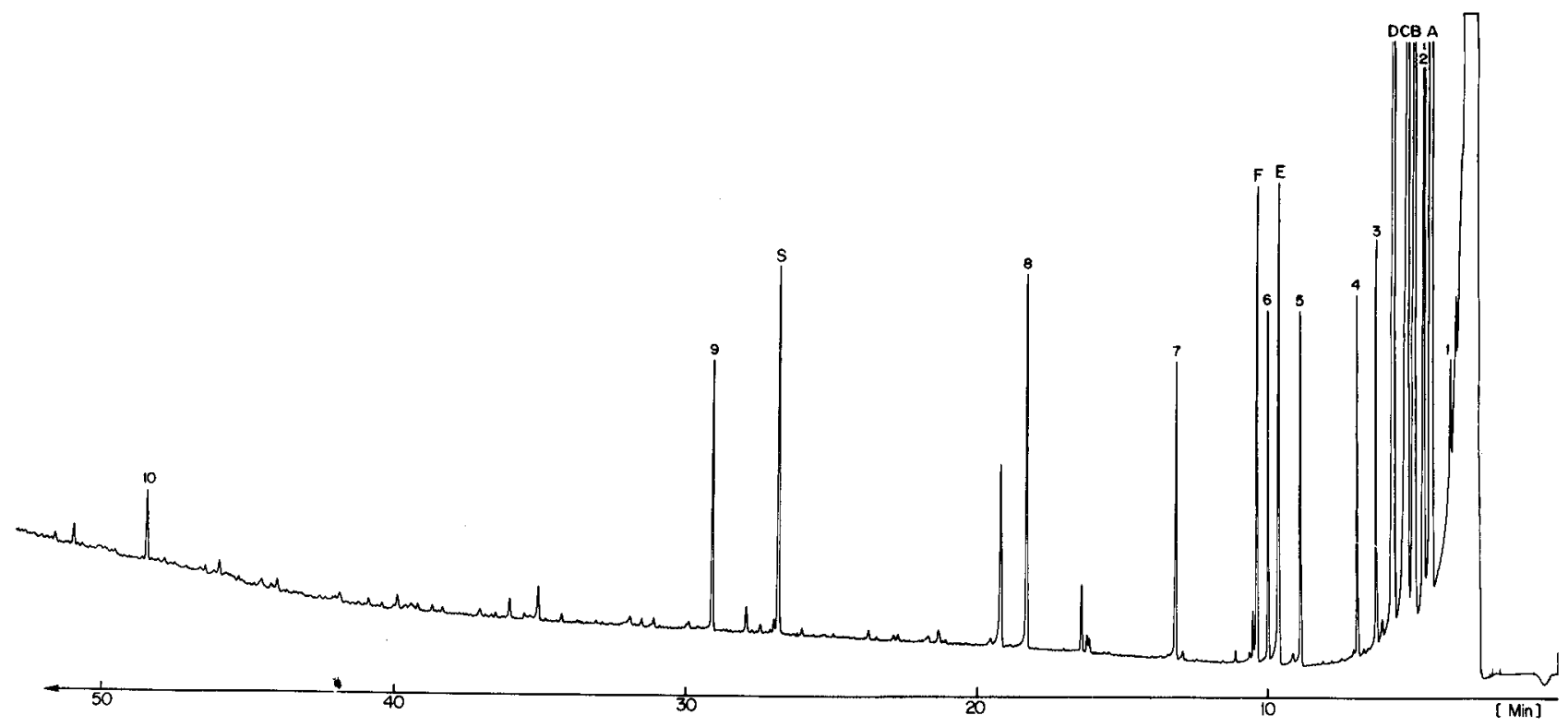

Fig. 2

- Gas-Chromatogramm eines mit Äthyljodid veresterten Standardsäuregemischs (5-50 $\mu \mathrm{g}$ je Säure verestert):

(1) Ameisen-, (2) Essig-, (3) Propion-, (4) Isobutter-, (5) Butter-, (6) 2-Methylbutter-, (7) Valerian-, (8) Capron-, (9) Capryl-, (10) Laurinsäure; $\mathrm{S}=$ interner Standard, $\mathrm{n}$-Tridecan.

Säule Ucon HB $5100,50 \mathrm{~m} \times 0.31 \mathrm{~mm} \mathrm{i}-\emptyset$, Injektion: $0.5 \mathrm{~mm}^{3}$ splitlos $/ 10 \mathrm{~s}$, Temp.-Progr.: $30-180^{\circ} \mathrm{C}, 3^{\circ} \mathrm{C} \min ^{-1}$, FID, Trägergas $\mathrm{H}_{2}$, $3 \mathrm{~cm}^{3} \mathrm{~min}^{-1}$. A-D Blindpeaks aus Reagenz und Nebenreaktionen $(\mathrm{A}=$ Äthyljodid, $\mathrm{B}=$ Äthanol $)$.

- Gas chromatogram of carboxylic acids esterified with ethyl iodide (5-50 $\mu \mathrm{g}$ per acid esterified):

(1) formic, (2) acetic, (3) propionic, (4) isobutyric, (5) butyric, (6) 2-methylbutyric, (7) valeric, (8) capronic, (9) caprylic, (10) lauric acid. $\mathrm{S}=$ internal standard, n-tridecane.

Column Ucon HB 5100, $50 \mathrm{~m} \times 0.31 \mathrm{~m}$ id, injection: $0.5 \mathrm{~mm}^{3}$ splitless $/ 10 \mathrm{~s}$, temp. program: $30-180{ }^{\circ} \mathrm{C}, 3{ }^{\circ} \mathrm{C} \mathrm{min}{ }^{-1}$, FI-detector, carrier gas $\mathrm{H}_{2}, 3 \mathrm{~cm}^{3} \mathrm{~min}^{-1} \mathrm{~A}-\mathrm{D}=$ blanks of reagent and products of side reactions $(\mathrm{A}=$ ethyl iodide, $\mathrm{B}=\mathrm{ethanol}$ ).

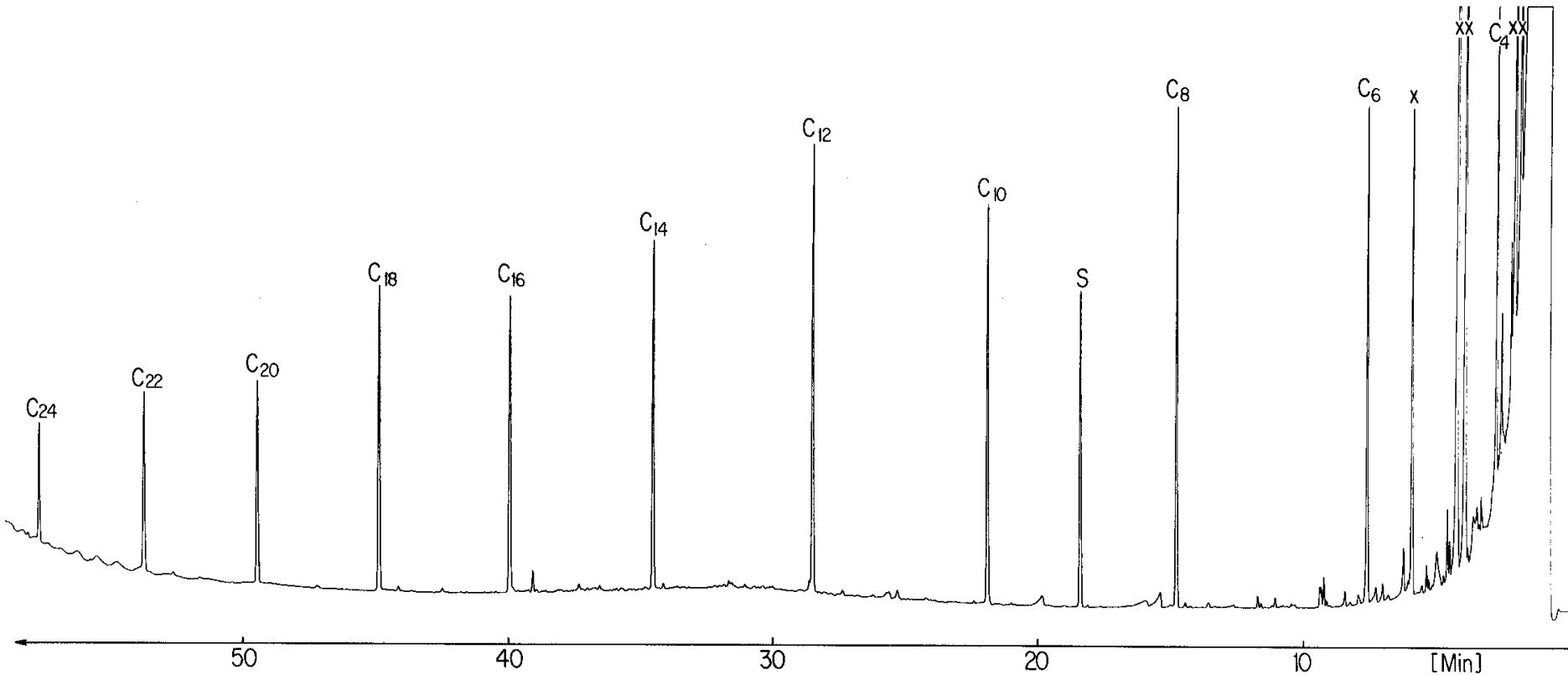

Fig. 3

- Gas-Chromatogramm eines mit Äthyljodid veresterten Standardgemischs der n-Monocarbonsäuren mit gerader C-Zahl zwischen $\mathrm{C}_{4}-\mathrm{C}_{24}$ $(10-50 \mu \mathrm{g}$ je Säure verestert):

Säule SE 54, $20 \mathrm{~m} \times 0.38 \mathrm{~mm}$ i- $\emptyset$, Injektion: $1 \mathrm{~mm}^{3}$ splitlos $/ 30 \mathrm{~s}$, Temp.-Progr.: $30-230^{\circ} \mathrm{C}, 4^{\circ} \mathrm{C} \mathrm{min}^{-1}$, FID, Trägergas $\mathrm{H}_{2}, 4.5 \mathrm{~cm}^{3} \mathrm{~min}^{-1}$, $\mathbf{x}=$ Blindpeaks aus Reagenz und Nebenreaktionen, $\mathrm{S}=$ interner Standard, $\mathrm{n}$-Tridecan.

- Gas chromatogram of even numbered $\left(\mathrm{C}_{4}-\mathrm{C}_{24}\right)$ n-monocarboxylic acids esterified with ethyl iodide (10-50 $\mu \mathrm{g}$ per acid esterified):

Column SE 54, $20 \mathrm{~m} \times 0.38 \mathrm{~mm}$ id, injection: $1 \mathrm{~mm}^{3}$ splitless $/ 30 \mathrm{~s}$, temp. program: $30-230{ }^{\circ} \mathrm{C}, 4{ }^{\circ} \mathrm{C} \mathrm{min}^{-1}$, FI-detector, carrier gas $\mathrm{H}_{2}$, $4.5 \mathrm{~cm}^{3} \mathrm{~min}^{-1}, \mathrm{x}=$ blanks of reagent and products of side reactions, $\mathrm{S}=$ internal standard, $\mathrm{n}$-tridecane. 


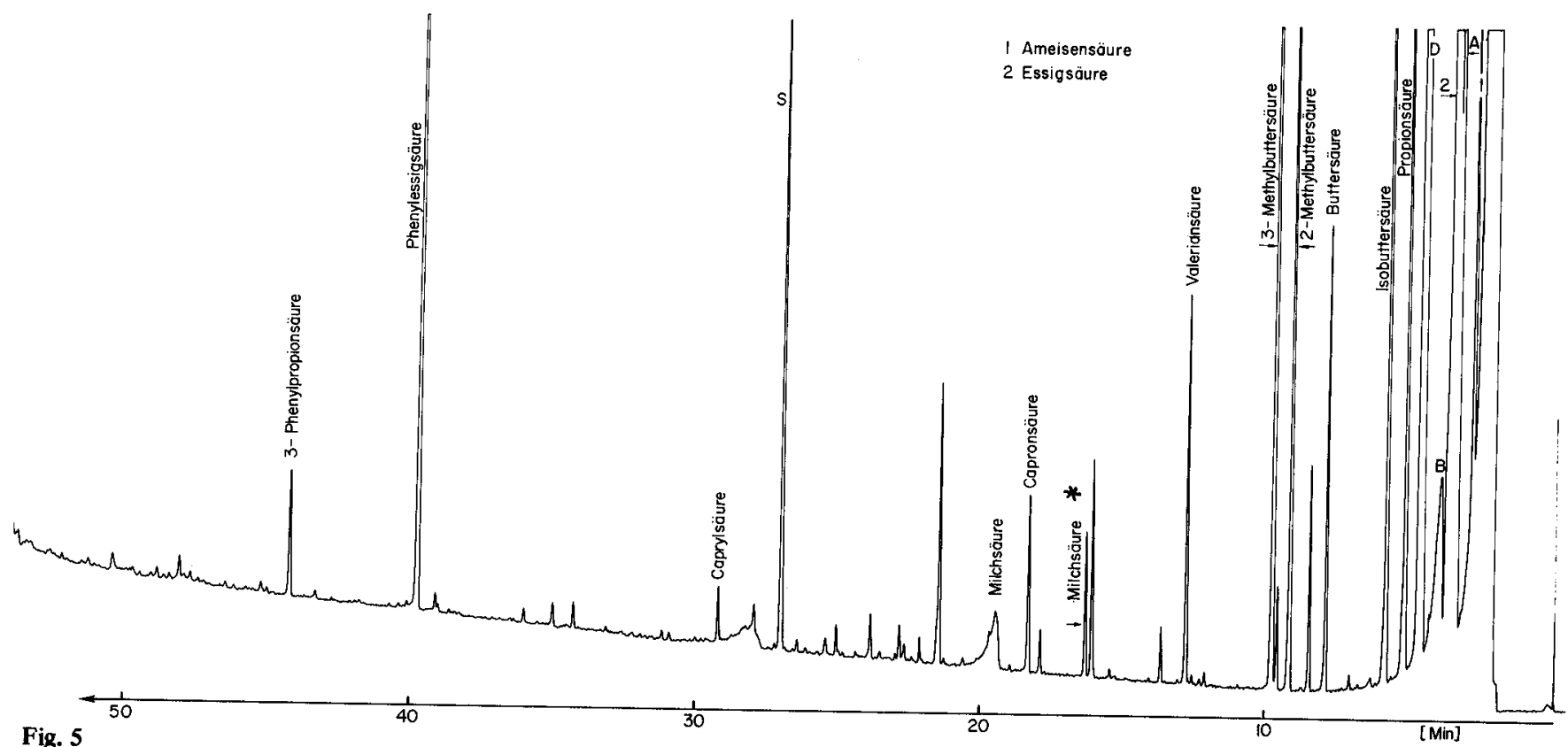

Fig. 5

- Gas-Chromatogramm der Carbonsäureäthylester eines Faulschlammwassers (aus Faulturm der ARA Opfikon, Zürich).

Ucon HB 5100-Säule, $50 \mathrm{~m} \times 0.31 \mathrm{~mm}$ i- $\emptyset$, Injektion: $0.5 \mathrm{~mm}^{3}$ splitlos $/ 10 \mathrm{~s}$, FID, Temp.-Progr.: $30-180^{\circ} \mathrm{C}, 3^{\circ} \mathrm{C} \mathrm{min}{ }^{-1}$, Trägergas $\mathrm{H}_{2}$, $3 \mathrm{~cm}^{3} \mathrm{~min}^{-1}, \mathrm{~A}-\mathrm{F}=$ Blindpeaks aus Reagenz und Nebenreaktionen, $\mathrm{S}=$ interner Standard, $\mathrm{n}$-Tridecan. Nicht beschriftete Peaks sind nicht identifiziert. Milchsäure* $=\alpha-\ddot{A}$ thoxy-äthylpropionat (s. Beispiel ,a“").

- Gas chromatogram of ethyl esters of carboxylic acids in the filtrate of digested sludge (sewage treatment plant Opfikon, Zürich). Column Ucon HB $5100,50 \mathrm{~m} \times 0.31 \mathrm{~mm}$ id, injection: $0.5 \mathrm{~mm}^{3}$ splitless $/ 10 \mathrm{~s}$, FI-detector, temp. program: $30-180^{\circ} \mathrm{C}, 3^{\circ} \mathrm{C} \mathrm{min}{ }^{-1}$, carrier gas $\mathrm{H}_{2}, 3 \mathrm{~cm}^{3} \mathrm{~min}^{-1} . \mathrm{A}-\mathrm{F}=$ blanks of reagent and side reactions, $\mathrm{S}=$ internal standard, $\mathrm{n}$-tridecane. Unnamed peaks not identified. Milchsäure* $=\alpha$-ethoxy ethyl propionate (cf. example "a").
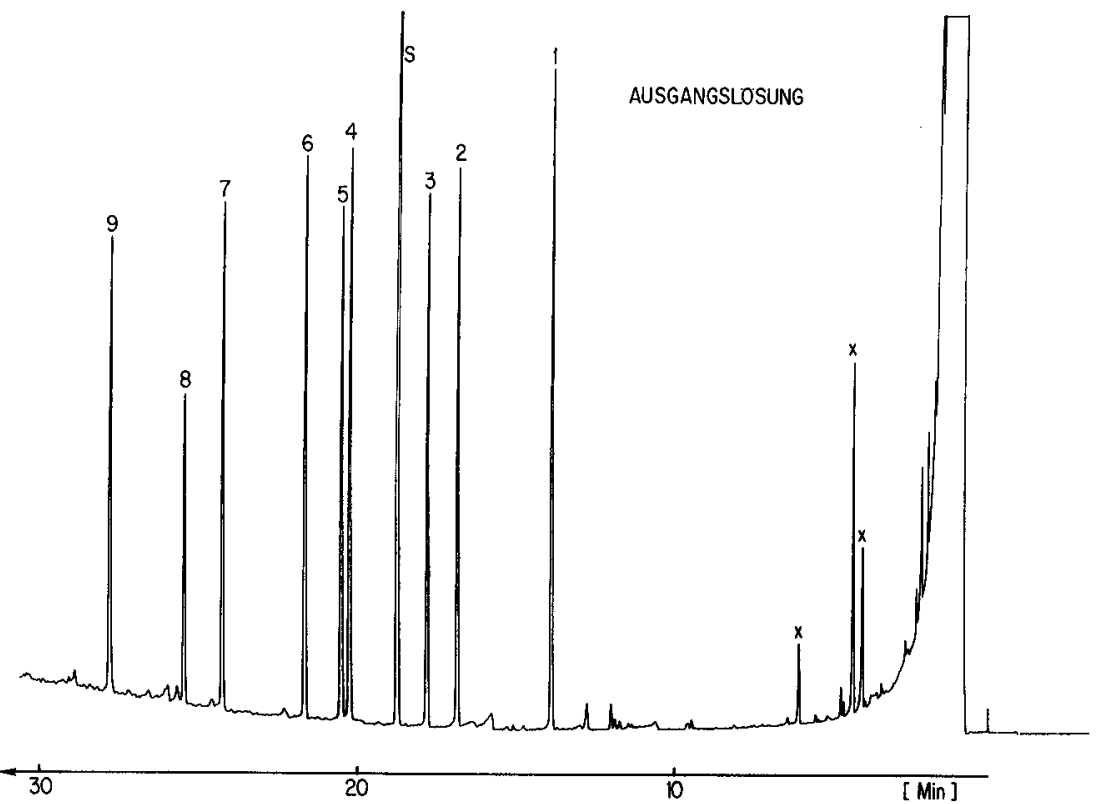

Fig. 6

- Gas-Chromatogramm einer mit Äthyljodid veresterten Phenylcarbonsäuremischung $(0.25 \cdots 0.75 \mu \mathrm{g}$ je Säure verestert):

(1) Benzoe-, (2) Phenylessig-, (3) 2-Phenylpropion-, (4) 2-Phenylbutter-, (5) 3-Phenylpropion-, (6) 3-Phenylbutter-, (7) 4-Phenylbutter-, (8) 4-Phenylvalerian-, (9) 5-Phenylvaleriansäure.

Säule SE $54,20 \mathrm{~m} \times 0.38 \mathrm{~mm}$ i- $\emptyset$, Injektion: $1.5 \mathrm{~mm}^{3}$ splitlos $/ 30 \mathrm{~s}$, Temp.Progr.: $30-150{ }^{\circ} \mathrm{C}, 4^{\circ} \mathrm{C} \mathrm{min}^{-1}$, FID, Trägergas $\mathrm{H}_{2}$, $4.5 \mathrm{~cm}^{3} \mathrm{~min}^{-1} . \mathrm{S}=$ interner Standard, $\mathrm{n}$-Tridecan, $\mathrm{x}=$ Blindpeaks aus Reagenz und Nebenreaktionen.

- Gas chromatogram of phenyl carboxylic acids esterified with ethyl iodide (2.5 to $7.5 \mu \mathrm{g}$ per acid esterified):

(1) benzoic, (2) phenyl acetic, (3) 2-phenyl propionic, (4) 2-phenyl butyric, (5) 3-phenyl propionic, (6) 3-phenyl butyric,

(7) 4-phenyl butyric, (8) 4-phenyl valeric, (9) 5-phenyl valeric acid.

Column SE $54,20 \mathrm{~m} \times 0.38 \mathrm{~mm}$ id, injection: $1.5 \mathrm{~mm}^{3}$ splitless $/ 30 \mathrm{~s}$, temp. program: $30-150{ }^{\circ} \mathrm{C}, 4{ }^{\circ} \mathrm{C} \mathrm{min}^{-1}$, FI-detector, carrier gas $\mathrm{H}_{2}, 4.5 \mathrm{~cm}^{3} \min ^{-1}, \mathrm{~S}=$ internal standard, $\mathrm{n}$-tridecane, $\mathrm{x}=$ blanks of reagent and products of side reactions. 
Tabelle I. Wiederholbarkeit und Richtigkeit der Veresterungsmethode (Prüfung an einem Standardsäuregemisch)

\begin{tabular}{|c|c|c|c|c|c|c|}
\hline \multirow[b]{2}{*}{ Versuchs-Nr. } & \multicolumn{5}{|c|}{$\mu \mathrm{g}$ Säure gefunden } & \multirow{2}{*}{$\begin{array}{l}\mu \mathrm{g} \text { Säure } \\
\text { theo- } \\
\text { retisch }\end{array}$} \\
\hline & 1 & 2 & 3 & 4 & MW & \\
\hline Essigsäure & 103 & 102 & 99 & 102 & 102 & 98 \\
\hline Propionsäure & 110 & 108 & 106 & 105 & 107 & 104 \\
\hline Isobuttersäure & 93 & 95 & 95 & 92 & 94 & 85 \\
\hline Buttersäure & 115 & 113 & 113 & 112 & 113 & 110 \\
\hline 2-Methylbuttersäure & 103 & 107 & 107 & 105 & 106 & 111 \\
\hline Valeriansäure & 82 & 81 & 82 & 80 & 81 & 82 \\
\hline
\end{tabular}

Tabelle II. Carbon-Säuren im Abwasser der Stadt Zürich (Schöpfprobe vom 25.5.1976)

\begin{tabular}{lrlr}
\hline Säure & $\mu \mathrm{g} / \mathrm{dm}^{3}$ & Säure & $\mu \mathrm{g} / \mathrm{dm}^{3}$ \\
\hline Ameisensäure & 538 & Benzoesäure & 170 \\
Essigsäure & 4490 & Bernsteinsäure & 79 \\
Propionsäure & 230 & Caprinsäure & 34 \\
Isobuttersäure & 29 & Phenylessigsäure & 91 \\
Buttersäure & 24 & 2-Phenylpropionsäure & 21 \\
2-Methylvaleriansäure & 20 & Laurinsäure & 110 \\
3-Methylvaleriansäure & 109 & Myristinsäure & 91 \\
Valeriansäure & - & Palmitinsäure & 354 \\
Milchsäure & 839 & Linolsäure & 174 \\
& & Ölsäure & 432 \\
Capronsäure & 62 & Stearinsäure & 188 \\
Caprylsäure & 36 & & \\
\hline
\end{tabular}

Tabelle III. Carbonsäuren im Faulschlammfiltrat

\begin{tabular}{lr}
\hline Säure & $\mathrm{mg} / \mathrm{dm}^{3}$ \\
\hline Phenylessigsäure & 7,9 \\
2-Phenylpropionsäure & 0,9 \\
Ameisensäure & 11,5 \\
Essigsäure & 73,3 \\
Propionsäure & 16,6 \\
Isobuttersäure & 15,3 \\
Buttersäure & 2,3 \\
2-Methylbuttersäure & 9,1 \\
3-Methylbuttersäure & 12,7 \\
Valeriansäure & 2,0 \\
Milchsäure & 2,1 \\
Capronsäure & 1,0 \\
Caprylsäure & 0,4 \\
\hline
\end{tabular}

Butter-, Capron-, Capryl-, Benzoe- und Laurinsäure) eine relative Standardabweichung von $15-20 \%$. Der Variationskoeffizient für Milchsäure und Bernsteinsäure betrug hingegen $\sim 50 \%$. Die große Streuung der Werte für diese beiden Säuren erklären wir mit der unvollständigen Bildung der entsprechenden Silbersalze im alkalischen Milieu. Als gas-chromatographische Information erhält man für Milchsäure zwei Signale, da die Reaktion des At thyljodids mit ihrem Silbersalz sowohl zum Ester (im Chromatogramm mit Milchsäure bezeichnet) als auch unter gleichzeitiger Reaktion mit der Hydroxylgruppe zum $\alpha$-Äthoxy-äthylpropionat (im Chromatogramm als Milchsäure* bezeichnet) führen kann. Für die Peakgruppe zwischen Stearinsäure und Di-(2-äthylhexyl)phthalat weisen massenspektroskopische Untersuchungen auf Steroide hin. Die Anwesenheit von Di-(2-äthylhexyl)phthalat (einem Weichmacher für synthetische Polymere) überrascht nicht und ist sicher auf die verbreitete Verwendung von Plastikmaterial in Haushalt, Labor und Industrie zurückzuführen. Alkylphthalate wurden bereits schon von Grob [16] sowohl in Trink(!)-, Oberflächen- und kontaminiertem Grundwasser nachgewiesen.

\section{b) Faulschlamm-Filtrat}

Fig. 5 zeigt das Gas-Chromatogramm eines Faulschlammfiltrates (aus Faulturm der ARA Opfikon, $\mathrm{ZH}$ ) von welchem $10 \mathrm{~cm}^{3}$ gemäß dem für kommunales Abwasser beschriebenen Vorgehen aufgearbeitet wurden.

Dominant ist Essigsäure mit $73 \mathrm{mg} / \mathrm{dm}^{3}$. Ameisen-, Propion-, Isobutter-, Butter-, 2-Methylbutter-, 3-Methylbutter-, Valerian-, Milch- und Phenylessigsäure liegen im Bereich von 2-16 ppm. Capron-, Capryl- und 2-Phenylpropionsäure wurden in Konzentrationen zwischen 0,4 und $1 \mathrm{ppm}$ nachgewiesen (Tab. III).

\section{c) Phenylcarbonsäuren}

Eine weitere Anwendung fand die Methode im Rahmen abbaukinetischer Untersuchungen [14] an Phenylcarbonsäuren unter den Bedingungen des OECD-ScreeningTests [15]. Fig. 6 zeigt das Gas-chromatogramm des Testsubstrates bei Versuchsbeginn.

\section{Dank}

Herrn Dr. K. Banholzer, Chef der Abteilung „Organische Chemie und Schmiermittel", EMPA, Dübendorf, danken wir für das zur Verfügung gestellte Massenspektrometer und Herrn Dr. $J$. $S$. Dolezal und Frl. E. Guyer für die Massenspektrometrischen Untersuchungen.

\section{Literatur}

[1] A. Ziolecki, E. Kwiatkoska, J. Chromatog. 80, 250 (1973).

[2] D. M. Ottenstein, D. A. Bartley, Analyt. Chem. 43, 952 (1971).

[3] J. Krupčik, J. Hrivñák, J. Janák, J. Chromatogr. Sci. 14, 4 (1976).

[4] K. Grob, G. Grob, Chromatographia 5, 3 (1972), ibd. 382 (1972).

[5] H. Schuchmann, Kontakte 2/75, 34 (1975).

[6] L. D. Metcalfe, A. A. Schmitz, R. J. Pelha, Analyt. Chem. 38, 514 (1966).

[7] S. Huwyler, Experientia 29, 735 (1973).

[8] J. A. Thompson, S. P. Markey, Analyt. Chem. 47, 1313 (1975).

[9] C. W. Gehrke, D. F. Goerlitz, Analyt. Chem. 35, 76 (1963).

[10] J. P. Riley, D. Taylor, Anal. Chim. Acta 46, 307 (1969).

[11] R. Kaiser, Chromatographie in der Gasphase. Band III, S. 133. Bibliographisches Institut, Mannheim (1961).

[12] H. A. Painter, M. Viney, J. of Biochemical and Microbiological Technol. and Engin. 1, 143 (1959).

[13] H. A. Painter, Chemistry and Industry 818 (1973).

[14] Untersuchung Biolog. Abteilung EAWAG.

[15] OECD: Pollution by detergents. Determination of the biodegradability of anionic synthetic surface active agents. Paris, 1971, S. 18.

[16] K. Grob et al., J. Chromatogr. 106, 299 (1975). 